\title{
Predicting stroke cause using imaging and RNA profiling
}

In more than $35 \%$ of patients with ischaemic stroke, the underlying cause is unknown, or cryptogenic. A study by Glen Jickling and colleagues describes a new approach to predicting stroke aetiology that combines brain imaging data with gene expression profiling, and could be used to improve treatment decision-making and stroke outcomes. "In cryptogenic strokes, opportunities to implement specific stroke prevention therapies can be missed," says Jickling.

The team of researchers, who are at the University of California at Davis, USA, had previously characterized RNA profiles of inflammatory and thrombotic genes that were differentially expressed in circulating leukocytes depending on stroke aetiology. A 41-gene expression profile was identified to distinguish lacunar from nonlacunar stroke, and a separate 40-gene expression profile was described that differentiated cardioembolic from arterial stroke. "One of the benefits of focusing on gene expression is that RNA is rapidly expressed in circulating leukocytes in response to stroke, well before protein," explains Jickling.

\section{$41 .$. RNA is rapidly expressed in circulating leukocytes in response to stroke... 77}

The current study involved 131 patients with cryptogenic stroke, who were compared with 149 patients with stroke of known aetiology. Vascular risk factors, sex, age, race and ethnicity were not significantly different between the groups.

Infarct location was determined on CT and MR angiography, and used to classify patients as having small deep infarcts (SDIs) or non-SDIs. These neuroimaging results were then used to determine which of the previously developed geneexpression profiles to screen for in RNA extracted from the patients' peripheral blood. The 41-gene expression profile was used to predict whether SDIs were lacunar or nonlacunar in origin, and the 40-gene expression profile was used to predict whether non-SDIs and nonlacunar SDIs had an arterial or cardioembolic aetiology.

Of the 131 cryptogenic stroke cases, 76 (58.0\%) were predicted to be cardioembolic and $24(18.3 \%)$ were predicted to be arterial-values that are consistent with previous epidemiological results. 15 (11.5\%) cryptogenic strokes were thought to be lacunar in origin, and $16(12.2 \%)$ remained of unknown aetiology.

Stroke subtypes were associated with specific clinical features, such as increased blood pressure in patients with stroke of predicted lacunar aetiology compared with those with predicted arterial or cardioembolic stroke. "Careful follow-up of patients with cryptogenic strokes may identify stroke causes that become apparent over time," says Jickling. The researchers highlight that further work is required to determine whether the stroke subgroups are associated with epidemiological or therapeutic differences.

Looking to future applications, Jickling says "the identified genes could be developed into a diagnostic test, such as are currently in clinical use for breast cancer and coronary artery disease."

Katie Kingwell

Original article Jickling, G. C. et al. Prediction of cardioembolic, arterial, and lacunar causes of cryptogenic stroke by gene expression and infarct location. Stroke doi:10.1161/STROKEAHA.111.648725 